\title{
Insulinoma unmasked by bariatric surgery
}

\begin{abstract}
Background: Hypoglycemia is a known complication of bariatric surgery and has been attributed to several mechanisms. A possible mechanism is hyperinsulinism due to islet cell hyperplasia (nesidioblastosis), and very rarely caused by insulinoma.

Clinical case: A 42-year-old morbidly obese man underwent biliopancreatic diversion, and after losing twenty kilograms of body weight four months later, he started to experience repeated episodes of severe hypoglycemia that were almost always induced by fasting; a blood sugar level as low as $1.6 \mathrm{mmol} /$ liter was confirmed with a history of several episodes of loss of consciousness.

Laboratory workup revealed the following: fasting blood sugar $2 \mathrm{mmol} /$ liter (NL 3.9-6.1), insulin $157 \mathrm{pmol} /$ liter (NL 43-193), C-Peptide $2521 \mathrm{pm} / \mathrm{liter}$ (NL 2601390), proinsulin $1100 \mathrm{pmol} /$ liter (NL 3-20). Screenings for serum sulfonylurea and meglitinides were negative. Based on the findings, we confirmed that the hypoglycemia was caused by inappropriate over secretion of endogenous insulin. A CT scan of the abdomen was performed and showed a $2 \times 1.6 \mathrm{~cm}$ tumor at the body of the pancreas. The mass was resected laparoscopically, and the patient's blood sugar subsequently normalized. He remained euglycemic for four years until the date of writing this report.
\end{abstract}

Conclusion: We report a very rare case of insulinoma that was unmasked by biliopancreatic diversion.
Volume 6 Issue 6 - 2018

Mohammed H AIQambar, Abdulaziz

AlWosaibei, Jamal AlSaeed, Wadei Elhakimi, Osama AISaif, Mohamed Elsammak, Hisham Musleh

Department of Medicine, Endocrinology section, King Fahd Specialist Hospital, Saudi Arabia

Correspondence: Mohammed H Alqambar, Endocrinologist, King Fahad Specialist Hospital, Po Box 13788 Tarout 31911, Saudi Arabia,Tel +966549 I30 55I,

Email ALQANBARM@YAHOO.COM

Received: October 18,2018 | Published: November 13, 2018

\section{Introduction}

With the tremendous increase in the rate of obesity, which is now considered an epidemic, the only durable form of management is surgical intervention what it is known as bariatric surgery.

Despite the favorable effects of bariatric surgery on obesityassociated morbidity and overall mortality, ${ }^{1}$ there has been mounting concern about severe hypoglycemia associated with this procedure. Hypoglycemia is a known complication of bariatric surgery and is attributed to several mechanisms, including hyperinsulinism due to islet cell hyperplasia but not insulinoma. ${ }^{2}$

We are reporting a case of insulinoma unmasked by bariatric surgery. To our knowledge there are only a few reported cases in the literature of confirmed insulinoma discovered after bariatric surgery. ${ }^{3-5}$

\section{Clinical case}

Our patient is a 42-year old morbidly obese man who underwent biliopancreatic diversion, and after losing twenty kilograms of body weight four months later, he started to have repeated episodes of severe hypoglycemia almost always induced by fasting with confirmed low blood sugar levels as low as $1.6 \mathrm{mmol} / \mathrm{liter}$ (NL 3.9 6.1) with a history of loss of consciousness several times. Two weeks before he underwent management, his hypoglycemia was persistent and he needed to be admitted to the hospital. He received continuous intravenous $10 \%$ dextrose to maintain his blood sugar just above 4 $\mathrm{mmol} /$ liter. His physical examination was unremarkable apart from morbid obesity with a BMI of $51 \mathrm{~kg} / \mathrm{m}^{2}$. His laboratory workup after stopping his intravenous dextrose for five minutes showed the following: blood sugar $2 \mathrm{mmol} / \mathrm{liter}$ (NL 3.9-6.1), insulin $157 \mathrm{pmol} /$ liter (NL 43-193), C-Peptide 2521 pm/liter (NL 260-1390), proinsulin
$1100 \mathrm{pmol} /$ liter (NL 3-20). Screenings for serum sulfonylurea and meglitinides were negative. His remaining blood work included the following: AM cortisol $282 \mathrm{nmol} /$ liter (NL 138-635) and kidney and liver function tests were all within normal limits. Based on these findings, we confirmed that the hypoglycemia was caused by inappropriate over secretion of endogenous insulin, and a MRI of the abdomen was performed (Figure 1), which showed a 2x1.6 cm tumor at the body of the pancreas. The patient underwent a laparoscopic partial pancreatectomy and his blood sugar normalized within minutes postoperatively. The pathological examination of the tumor revealed a $1.5 \mathrm{~cm}$ low grade islet cell neoplasm consistent with insulinoma (Figure 2).

The patient remained euglycemic for 4 years after surgery until the time of writing this report.

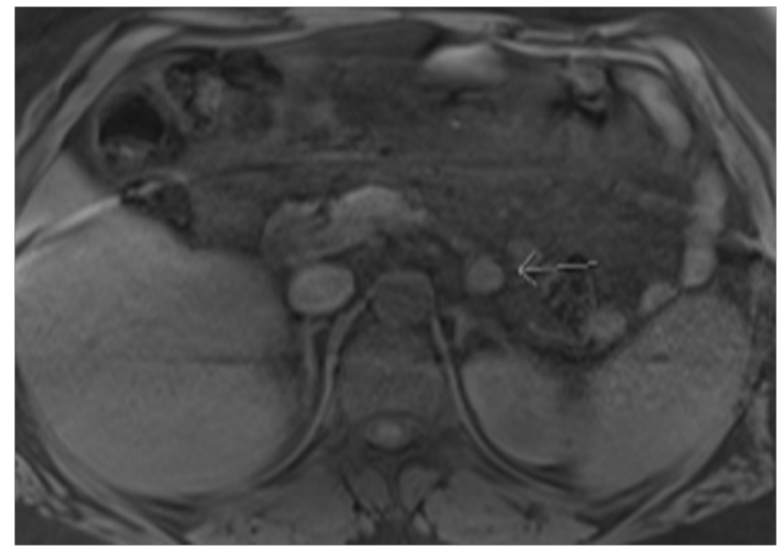

Figure I MRI: tumor at the body of pancreas. 


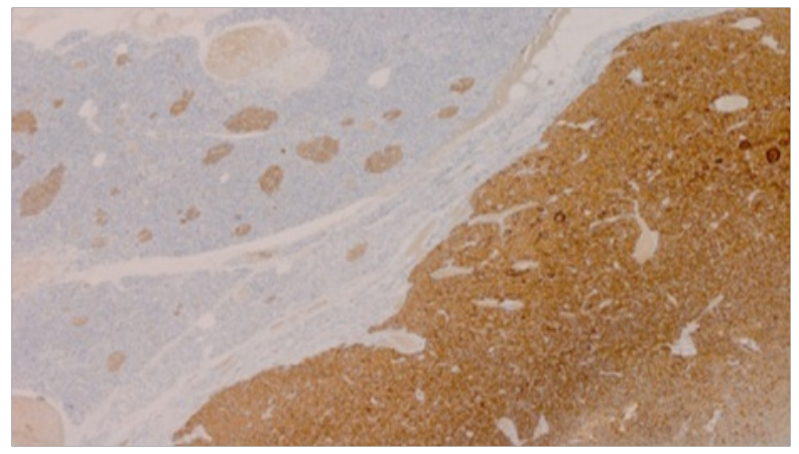

Figure 2 Synaptophysin stain in tumor cells and islets.

\section{Discussion}

In particular instances, patients who have undergone bariatric surgery experience postprandial symptoms related to dysglycemia, and several mechanisms have been described. Dumping syndrome is probably the most prevalent problem; the patient usually feels diaphoresis, weakness, dizziness, and flushing while infrequently experiencing neuroglycopenia. Another etiology is islet cell hyperplasia (nesidioblastosis) in which glucose levels can significantly drop and patients commonly present with neuroglycopenic symptoms including cognitive impairment, behavioral changes, confusion, seizures and even coma. The pathogenesis of nesidioblastosis in patients who have undergone gastric bypass surgery is still unclear. A theoretical possibility is that in obese persons with insulin resistance, adaptive beta-cell hypertrophy develops and causes hypoglycemia after insulin sensitivity is improved by the surgically induced weight loss. Service et al. argued against this idea as a mechanism by showing that the islets in obese control subjects without gastric bypass are of normal size. ${ }^{4}$ Moreover, there is no association between non-surgically induced weight loss and endogenous hypoglycemia or nesidioblastosis. Cummings et al. hypothesized that an increase in glucagon-like peptide-1 (GLP-1) concentration may be responsible for the islet cell expansion ${ }^{6}$ and this mechanism explains why $40 \%$ of patients who had nesidioblastosis had undergone gastric bypass surgery. ${ }^{7}$ It is very uncommon to have an insulinoma manifest after bariatric surgery, and only very few cases have been reported in the literature. Our patient started to have symptoms of hypoglycemia 4 months post-surgery, which is too early for nesidioblastosis to develop, and it typically causes postprandial rather than fasting hypoglycemia.
We hypothesize that the severe insulin resistance due to morbid obesity was compensating for hyperinsulinemia before surgery, which protected him from having any hypoglycemic symptoms. After losing a remarkable amount of weight postoperatively, insulin sensitivity improved and he started to experience progressive hypoglycemic symptoms.

\section{Conclusion}

We report a very rare case of insulinoma that was unmasked by biliopancreatic diversion, and we believe such an etiology should be considered in the workup of post-bariatric hypoglycemia.

\section{Acknowledgments}

None.

\section{Conflict of interest}

The author declares there is no conflict of interest.

\section{References}

1. Sjostrom L, Narbro K, Sjostrom CD, et al. Effects of bariatric surgery on mortality in Swedish obese subjects. N Engl J Med. 2007;357(8):741-752.

2. Foster-Schubert KE. Hypoglycemia complicating bariatric surgery: Incidence and mechanisms. Curr Opin Endocrinol Diabetes Obes. 2011;18(2):129-133.

3. Zagury L, Moreira RO, Guedes EP, et al. Insulinoma misdiagnosed as dumping syndrome after bariatric surgery. Obes Surg. 2004;14(1):120 123.

4. Duff JM, Ghayee HK, Weber M, et al. Delayed Imaging Presentation of a Symptomatic Insulinoma After Bariatric Surgery. J Gastrointest Surg. 2017;21(2):412-414.

5. Christopher M. Insulinoma After Bariatric Surgery: Diagnostic Dilemma and Therapeutic Approaches. Obes Surg. 2016;26(4):874-881.

6. Cummings DE. Gastric bypass and nesidioblastosis: too much of a good thing for islets? N Engl J Med. 2005;353(3):300-302.

7. Carpenter T, Trautmann ME, Baron AD. Hyperinsulinemic hypoglycemia with nesidioblastosis after gastric-bypass surgery. $N$ Engl $\mathrm{J} \mathrm{Med}$. 2005;353(20):2192-2194. 